\title{
Prevalence and Predictive Factors of Fecal Incontinence
}

\author{
Hye-Won Kang, Hye-Kyung Jung, ${ }^{*}$ Kyoung-Joo Kwon, Eun-Mi Song, Ju-Young Choi, Seong-Eun Kim, Ki-Nam Shim and \\ Sung-Ae Jung
}

Department of Internal Medicine, Ewha Medical Research Institute, Ewha Womans University School of Medicine, Seoul, Korea

\section{Background/Aims}

Most previous epidemiologic studies about fecal incontinence were performed in specific populations in Korea. We aimed to estimate the prevalence and predictive factors of fecal incontinence in adult Korean population, both men and women aged 20 years and over.

\section{Methods}

Subjects who had undergone medical check-up for health screening were enrolled. They completed the structured questionnaires, including demographics, gastrointestinal symptoms, medical and social histories, and also about their bowel habits. Logistic regression models were constructed to identify the predictive factors for having fecal incontinence.

\section{Results}

Among the total of 1,149 subjects (mean age, $44.8 \pm 10.2$ years; 648 males), the overall prevalence of fecal incontinence was $6.4 \%$, while the older group ( $>50$ years old) showed the higher prevalence than the younger group ( $\leq 50$ years old) $(10.4 \%$ vs $4.9 \%, P=0.001)$ without gender difference. Most patients had mild fecal incontinence in $78.4 \%$. By multivariate analysis, old ages (Odd ratio $[\mathrm{OR}], 3.1 ; 95 \%$ confidence interval $[\mathrm{Cl}], 1.9-5.2 ; P<0.001)$, watery stool $(\mathrm{OR}, 2.8 ; 95 \% \mathrm{Cl}$, 1.5-4.9; $P=0.001)$ and functional diarrhea $(\mathrm{OR}, 2.7 ; 95 \% \mathrm{Cl}, 1.4-5.4 ; P=0.004)$ were found to be independent predictors for fecal incontinence.

\section{Conclusions}

The prevalence of fecal incontinence in Korean adults was 6.4\%, and it was significantly more prevalent in older people without any gender difference. Aging and diarrhea were independent predictive factors of fecal incontinence. Therefore, proper control of the bowel pattern would lead to the prevention of fecal incontinence.

\section{(J Neurogastroenterol Motil 2012;18:86-93)}

\section{Key Words}

Epidemiology; Fecal incontinence; Prevalence; Risk factors

Received: September 8, 2011 Revised: October 24, 2011 Accepted: November 3, 2011

(c) This is an Open Access article distributed under the terms of the Creative Commons Attribution Non-Commercial License (http://creativecommons. org/licenses/by-nc/3.0) which permits unrestricted non-commercial use, distribution, and reproduction in any medium, provided the original work is properly cited.

*Correspondence: Hye-Kyung Jung, MD, PhD

Division of Gastrointestinal Diseases, Department of Internal Medicine, Ewha Womans University Mokdong Hospital, 911-1

Mok-dong, Yangcheon-gu, Seoul 158-710, Korea

Tel: +82-2-2650-2874, Fax: +82-2-2655-2874,E-mail: junghk@ewha.ac.kr

Financial support: None.

Conflicts of interest: None. 


\section{Introduction}

Fecal incontinence, which is defined as the accidental loss of solid or liquid stool, is known as a condition which would have a significant impairment on the patient's physical and psychological status. ${ }^{1-5}$ In population-based study of the United States, the estimated prevalence of fecal incontinence range from $2.2 \%{ }^{5}$ to as high as $24 \%$, with most estimates within the range of $7 \%-12 \%{ }^{1,7}$

Fecal incontinence seems to reduce the work productivity, narrow the physical activity, and harsh self images. It has a significant impact on quality of life (QOL) that may include embarrassment, social stigmatization, depression, and anxiety. ${ }^{8} \mathrm{QOL}$ of these patients are known to be negatively correlated with the severity of fecal incontinence, especially in older females, which implies that delayed treatments for this condition could gradually bring results of poorer QOL., ${ }^{9,10}$

Furthermore, fecal incontinence would impose high cost on the patient and the community. ${ }^{2-4}$ Fecal incontinence is one of the most common reasons for admittance to nursing homes, ${ }^{1-6}$ and is becoming the second most common cause for institutionalization of the elderly people in the USA. Americans also spend more than 400 million dollars per year for buying adult diapers. ${ }^{2}$ However, its importance has been underestimated because those patients tend to avoid reporting their symptoms. Surveys suggest that only $10 \%-30 \%$ of people with fecal incontinence have discussed this issue with their physician. ${ }^{8,11}$ Therefore, diagnosis and proper managements are often delayed. ${ }^{2}$

As elderly people in Korean society are increasing, fecal incontinence might also be an important issue in terms of the people's QOL and the health care costs. However, until now, there were several limited studies with fecal incontinence in Korea.

Therefore, we aimed to estimate the prevalence of fecal incontinence and also identify predictive factors of fecal incontinence in Korean adult population aged 20 years and over.

\section{Materials and Methods}

\section{Subjects}

The study population was consisted of 1,149 subjects who had come to Health Promotion Center in Ewha Womans University Mokdong Hospital from September 2008 to December 2009. Health Promotion Center is operated for regular medical check-up. Two-thirds of the study subjects underwent a group medical examination for worker's health screening and one-third paid for by themselves. Every subject filled out our structured questionnaires asking about their symptoms after giving the written informed consent. Anyone who visited our center in that period was not excluded in this study. We got an approval from institutional review board before starting this study.

\section{Questionnaires and Laboratory Tests}

To evaluate the prevalence of fecal incontinence, we used the validated Korean version of bowel disease questionnaire (K-BDQ) following the Rome III criteria. Previous testing has shown the BDQ to be reliable, with a median kappa statistic of 0.78 for symptom items (range, 0.52-1.00). ${ }^{12}$ The K-BDQ is composed of 30 items distinguishing several functionally diverse bowel diseases such as irritable bowel syndrome (IBS), functional dyspepsia, functional bloating, functional constipation, functional diarrhea, and fecal incontinence. Intelligibility of the questionnaire was maximized by adhering to an assumed sixth grade reading level and was supported by the high literacy rate. The median kappa value of reliability test for K-BDQ was 0.74 (0.36-1.00), which is suggestive of good correlation. ${ }^{13}$ The symptoms severity and frequency of self-reporting fecal incontinence were assessed. Each stool consistency was classified into hard (form 1 and 2), normal (form 3,4 and 5) and watery (form 6 and 7) patterns by Bristol stool scale (BSS).

Also, questions were designed to evaluate bowel habit dysfunction (self-reported bowel habit, frequency, incomplete evacuation, consistency, straining, self-digitation and feeling of anal blockage). The somatic symptom checklist (SSC) was used to measure the degree of somatization based on 17 items, such as headache, fatigue, nervousness and so on. ${ }^{14}$

Information about the possible risk factors was as follows:

(1) Demographics: age and sex

(2) Body mass index (BMI): height and weight measured by body composition analyzer (InBody 720, BioSpace ${ }^{\circledR}$, Seoul, Korea). BMI was calculated as weight divided by the square of height.

(3) Co-morbidities: hypertension, diabetes, cerebrovascular disease, dementia, malignancy, depression and history of cholecystectomy. These factors were defined as below.

A. Hypertension - medical history of diagnosis of hypertension by doctors

B. Diabetes - medical history of diagnosis of diabetes by doctors 
C. Cerebrovascular disease - medical history of cerebrovascular accidents

D. Dementia - diagnosed by doctor or if some medications have been taken.

E. Malignancy - history of any cancer or currently active stage of cancer

F. Depression - medical history or self-reported depressed mood at least more than once a week

G. Cholecystectomy - history of open or laparoscopic cholecystectomy

\section{Definitions}

Fecal incontinence was defined as events of recurrent uncontrolled passage of fecal materials at least once during the recent 3 months. Subjects fulfilling the criteria of fecal incontinence were classified into the subgroups of hard, normal and watery type by BSS, based on their daily stool type. The severity of fecal incontinence was defined by the amount of fecal materials. The mild fecal incontinence was defined by small amount of stool passing (much smaller than usual) which has stained one's underwear, while severe fecal incontinence was defined when the profound amount (more than usual) of passed stool has even stained the outerwear. In cases of 'moderate,' the fecal amount might be smaller than the usual defecation, but the patient would need to change the underwear because of fecal material. Other functional gastrointestinal disorders were defined by the Rome III criteria. ${ }^{15}$

\section{Statistical Methods}

Statistical analysis was performed using the SPSS version 16.0 (SPSS Inc., Chicago, IL, USA). The continuous variables were expressed as mean $\pm \mathrm{SD}$. Univariate associations were analyzed using the Student's $t$ test for continuous variables, the Pearson's chi-square test and Fisher exact test for categorical variables. SSC was calculated as the mean of the frequency score and severity score in each item. The severe SSC was defined as the SSC equal as or higher than 1.

All variables significantly associated with fecal incontinence $(P<0.05$, two tailed) were included in the multivariate logistic regression models. Results were presented by odd ratios (OR) with $95 \%$ confidential intervals $(\mathrm{CI})$.

\section{Results}

\section{Clinical Characteristics of Subjects}

Among a total of 1,149 subjects, $56.4 \%$ were male subjects. Their mean age was $44.8 \pm 10.2$ years, ranging from 20 to 82 years old. Table 1 summarizes the characteristics of subjects. The overall prevalence of hypertension and diabetes was $6.8 \%$ and $3 \%$, respectively. The prevalence of cerebrovascular disease, dementia and cancer resulted as $6.5 \%, 2.4 \%$ and $15.3 \%$, respectively. The proportion of obese people whose BMI was higher than $25 \mathrm{~kg} / \mathrm{m}^{2}$ by Asian-Pacific criteria ${ }^{16}$ was $11.3 \%$, and mean value of BMI was $23.7 \pm 3.1 \mathrm{~kg} / \mathrm{m}^{2}$. Eleven subjects had taken cholecystectomy. The most prevalent BSS pattern was the 'normal' subgroup with $79.7 \%$, while $16.1 \%$ was 'watery' and $4.2 \%$ was 'hard'. Following the Rome III criteria, the prevalence of IBS, bloating, functional dyspepsia and functional constipation

Table 1. Characteristics Associated With Fecal Incontinence

\begin{tabular}{lcc}
\hline & $\begin{array}{c}\text { Fecal incontinence } \\
(\mathrm{n}=74)\end{array}$ & $\begin{array}{c}\text { Controls } \\
(\mathrm{n}=1,075)\end{array}$ \\
\hline Age (mean $\pm \mathrm{SD}, \mathrm{yr})$ & $49.0 \pm 10.6$ & $44.5 \pm 10.1$ \\
Age above $50 \mathrm{yr}(\mathrm{n}[\%])$ & $33(44.6)$ & $284(26.4)$ \\
Female gender $(\mathrm{n}[\%])$ & $34(45.9)$ & $467(43.4)$ \\
$\mathrm{BMI} \geq 25 \mathrm{~kg} / \mathrm{m}^{2}(\mathrm{n}[\%])$ & $9(12.2)$ & $121(11.3)$ \\
Underlying diseases (n [\%]) & & \\
Hypertension & $5(6.8)$ & $73(6.8)$ \\
Diabetes mellitus & $5(6.8)$ & $30(2.8)$ \\
CVA & $21(28.4)$ & $393(36.6)$ \\
Dementia & $2(2.7)$ & $26(2.4)$ \\
Malignancy & $11(14.9)$ & $165(15.3)$ \\
Cholecystectomy & $2(2.7)$ & $9(0.8)$ \\
Stool consistency (n [\%]) & & \\
Watery stool & $29(39.2)$ & $156(14.5)$ \\
Hard stool & $2(2.7)$ & $46(4.3)$ \\
Functional bowel diseases (n [\%]) & & \\
IBS & $15(20.3)$ & $124(11.5)$ \\
Functional constipation & $15(20.3)$ & $119(11.1)$ \\
Functional diarrhea & $18(21.2)$ & $56(5.3)$ \\
Functional bloating & $5(6.8)$ & $96(8.9)$ \\
Functional dyspepsia & $17(23.0)$ & $173(16.1)$ \\
Psychological problems (n [\%]) & & \\
Self reported depressed mood & $21(28.4)$ & $191(17.8)$ \\
Severe somatization & $23(31.1)$ & $158(14.8)$ \\
\hline
\end{tabular}

BMI, body mass index; CVA, cerebrovascular disease; Watery stool, Bristol Stool Scale form of 6 or 7; IBS, irritable bowel syndrome.

Self reported depressed mood, experiencing symptoms at least once a week; Severe somatization, defined as same or above 1 of somatization symptom scores. 
was $12.1 \%, 8.8 \%, 16.5 \%$ and $7.4 \%$, respectively. The proportion of subjects who reported to experience feelings of depression was $37.5 \%$ in more than once a month and $18.5 \%$ in at least once a week. The proportion of subjects with severe somatization was $15.8 \%$.

\section{Prevalence of Fecal Incontinence}

The prevalence of fecal incontinence was $6.4 \%$ (74 of 1,149), and it showed significantly increasing trend by aging $(P<0.05)$ (Fig. 1). Results from subjects older than 50 years old were significantly higher than those from the younger ones $(10.4 \%$ vs $4.9 \%, P=0.001)$. No significant difference was observed between men and women $(6.2 \%$ vs $6.8 \%, P=0.717)$. Among the subjects above 50 years old, the proportion of fecal incontinence in women was slightly higher than that in men, however, it did not reach the statistical significance $(12.4 \%$ vs $8.9 \%, P=0.355)$.

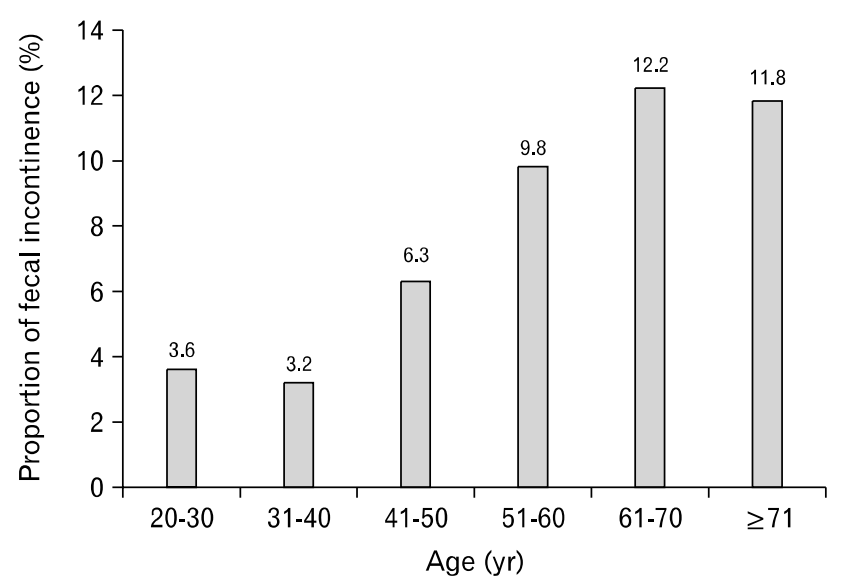

Figure 1. Prevalence of fecal incontinence. The proportion of fecal incontinence is increasing by aging.
Mild incontinence was reported in $78.4 \%$, while severe fecal incontinence was $1.4 \%$ (Fig. 2). Among 74 subjects with fecal incontinence, 2 subjects experienced it more than once a week. Ten subjects experienced fecal incontinence for 2-3 days per month, while it occurred almost once a month to another 10 subjects. About $58.1 \%$ of the subjects with incontinence had normal stool as their daily stool pattern. However, the proportion of subjects in incontinence group who had watery stool as their daily stool pattern was significantly higher than that of the control group (39.2\% vs $14.5 \%, P<0.001)$ (Fig. 3$)$.

\section{Assessment of Predictors for Fecal Inconti- nence by Univariate and Multivariate Analy- sis}

By univariate analysis, old age, watery stool, IBS, functional constipation, functional diarrhea, self reported depressed mood

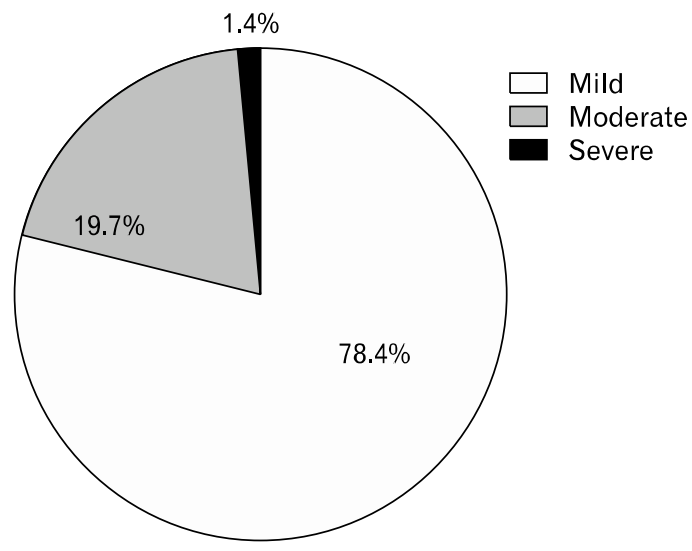

Figure 2. Severity of fecal incontinence. Severe fecal incontinence with large amount of leakage is $1.4 \%$, and mild fecal incontinence with small amount of fecal contents staining just innerwear is $78.4 \%$.
A

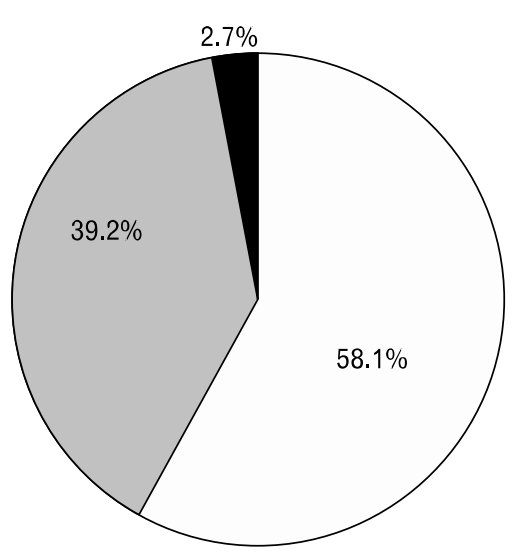

B

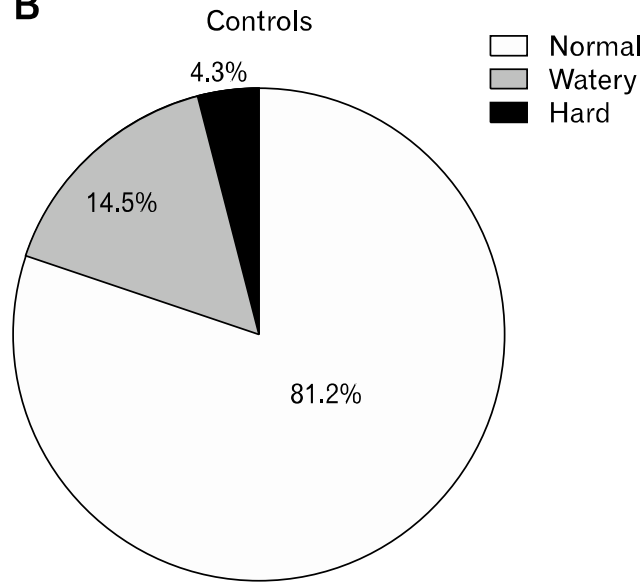

Figure 3. Stool consistencies in fecal incontinence group and control. (A) In fecal incontinence group, normal stool was the most prevalent type of their daily stool form (normal stool 58.1\%, watery stool $39.2 \%$ and hard stool $2.7 \%$ ). (B) In control group, the most frequent stool consistency was normal form (81.2\%), however, the proportion of watery stool in fecal incontinence group was significantly higher than that of control group (39.2\% vs $14.5 \%, P<0.001)$. 
and severe somatization were proved to be significant predictive factors for fecal incontinence (Table 2). In moderate to severe fecal incontinence, only severe somatization was the significant predictive factor (OR, 5.1; 95\% CI, 1.7-15.7, $P=0.008$ ). There was no correlation between fecal incontinence and other factors, such as hypertension, diabetes, CVA, dementia, cancer, obesity, cholecystectomy, smoking, functional bloating and functional

Table 2. Univariate Analysis of Predictive Factors for Fecal Incontinence

\begin{tabular}{lccc}
\hline & OR & $95 \%$ CI & $P$-value \\
\hline Age above 50 yr & 2.2 & $1.4-3.6$ & 0.001 \\
Female gender & 1.1 & $0.7-1.8$ & 0.717 \\
Obesity & 1.2 & $0.8-2.0$ & 0.370 \\
Co-morbidities & & & \\
$\quad$ Hypertension & 1.0 & $0.4-2.5$ & 1.000 \\
$\quad$ Diabetes mellitus & 2.5 & $0.9-6.7$ & 0.069 \\
CVA & 1.5 & $0.7-3.5$ & 0.324 \\
Dementia & 1.1 & $0.3-4.8$ & 0.700 \\
$\quad$ Malignancy & 0.9 & $0.5-1.8$ & 0.307 \\
$\quad$ Cholecystectomy & 3.3 & $0.7-15.5$ & 0.155 \\
Stool consistency & & & \\
$\quad$ Watery stool & 3.8 & $2.3-6.2$ & $<0.001$ \\
$\quad$ Hard stool & 0.6 & $0.1-2.6$ & 0.764 \\
Functional bowel diseases & & & \\
$\quad$ IBS & 2.0 & $1.1-3.5$ & 0.040 \\
Functional constipation & 2.0 & $1.1-3.7$ & 0.024 \\
$\quad$ Function diarrhea & 4.8 & $2.7-8.7$ & $<0.001$ \\
$\quad$ Functional bloating & 1.3 & $0.5-3.2$ & 0.672 \\
$\quad$ Functional dyspepsia & 1.6 & $0.9-2.7$ & 0.144 \\
Psychological problems & & & \\
$\quad$ Self reported depressed mood & 1.8 & $1.1-3.1$ & 0.029 \\
Severe somatization & 2.6 & $1.6-4.4$ & 0.001 \\
\hline
\end{tabular}

CVA, cerebrovascular disease; Obesity, body mass index same or above 25 $\mathrm{kg} / \mathrm{m}^{2}$; Watery stool, Bristol Stool Scale form of 6 or 7; IBS, irritable bowel syndrome.

Self reported depressed mood, experiencing symptoms at least once a week; Severe somatization, defined as same or above 1 of somatization symptom scores. dyspepsia.

Statistically significant predictors for fecal incontinence by multivariate analysis were old age (OR, 3.1; 95\% CI, 1.9-5.2; $P$ $<0.001)$, watery stool (OR, 2.8; 95\% CI,1.5-4.9; $P=0.001)$ and functional diarrhea $(\mathrm{OR}, 2.7 ; 95 \% \mathrm{CI}, 1.4-5.4 ; P=0.004)$ (Table 3).

In subjects above 50 years old, watery stool (OR, 4.1; 95\% CI, 1.8-9.6; $P=0.002)$, functional constipation (OR, 3.1; $95 \%$ $\mathrm{CI}, 1.2-7.8 ; P=0.025)$, functional diarrhea $(\mathrm{OR}, 6.7 ; 95 \% \mathrm{CI}$, 2.4-18.7; $P=0.001)$ and severe somatization $(\mathrm{OR}, 2.8 ; 95 \% \mathrm{CI}$, $1.3-6.1 ; P=0.016)$ were significant predictive factors for fecal incontinence. However, in subjects younger than 50 years old, 4 factors including watery stool $(\mathrm{OR}, 4.4 ; 95 \% \mathrm{CI}, 2.3-8.4 ; P<$ $0.001)$, functional diarrhea $(\mathrm{OR}, 4.8 ; 95 \% \mathrm{CI}, 2.3-10.1 ; P<$ $0.001)$, IBS (OR, 2.4; 95\% CI, 1.2-5.0; $P=0.019)$ and severe somatization (OR, 2.2; 95\% CI, 1.1-4.6; $P=0.038$ ) were significantly correlated with fecal incontinence (Table 4).

\section{Discussion}

We documented that the prevalence of fecal incontinence in Korean who visited for regular check-up at Health Promotion Center was $6.4 \%$. It was relatively lower than those of other previous Korean results. Joh et $\mathrm{al}^{17}$ reported that the prevalence of fecal incontinence in the elderly people was $15.5 \%$. Although the

Table 3. Multivariate Analysis of Predictive Factors for Fecal Incontinence

\begin{tabular}{lccr}
\hline & Adjusted OR & $95 \% \mathrm{CI}$ & $P$-value \\
\hline Age above $50 \mathrm{yr}$ & 3.1 & $1.9-5.2$ & $<0.001$ \\
Watery stool & 2.8 & $1.5-4.9$ & 0.001 \\
Functional diarrhea & 2.7 & $1.4-5.4$ & 0.004 \\
\hline
\end{tabular}

Watery stool, Bristol Stool Scale form of 6 or 7.

Table 4. Predictive Factors of Fecal Incontinence According to Aging

\begin{tabular}{|c|c|c|c|c|c|c|}
\hline & \multicolumn{3}{|c|}{ Younger age $(\leq 50 \mathrm{yr})$} & \multicolumn{3}{|c|}{ Older age $(>50 \mathrm{yr})$} \\
\hline & OR & $95 \% \mathrm{CI}$ & $P$-value & OR & $95 \% \mathrm{CI}$ & $P$-value \\
\hline Watery stool & 4.4 & $2.3-8.4$ & $<0.001$ & 4.1 & $1.8-9.6$ & 0.002 \\
\hline IBS & 2.4 & $1.2-5.0$ & 0.019 & 1.7 & $0.6-5.4$ & 0.312 \\
\hline Functional constipation & 1.8 & $0.8-3.9$ & 0.220 & 3.1 & $1.2-7.8$ & 0.025 \\
\hline Functional diarrhea & 4.8 & $2.3-10.1$ & $<0.001$ & 6.7 & $2.4-18.7$ & 0.001 \\
\hline Self reported depressed mood & 1.5 & $0.7-3.1$ & 0.296 & 2.3 & $1.0-5.0$ & 0.060 \\
\hline Severe somatization & 2.2 & $1.1-4.6$ & 0.038 & 2.8 & $1.3-6.1$ & 0.016 \\
\hline
\end{tabular}

Watery stool, Bristol Stool Scale form of 6 or 7; IBS, irritable bowel syndrome. 
definition of fecal incontinence was same with our study, the mean age of study population was $73.6 \pm 6.8$ years with higher female proportion. In the present study, the prevalence of fecal incontinence among the subjects older than 50 years old was $10.4 \%$. Our result is lower than that of Caucasian population based study, which showed the prevalence of $15.3 \%$ in responders whose median age was 65 years with a range of 50-91 years. ${ }^{3}$ In another Korean study conducted in subjects with diabetes in referral hospitals, the prevalence of fecal incontinence was $10.8 \%$ in men and $11.4 \%$ in women, which was higher than our result. ${ }^{18}$ This study applied the same definition of fecal incontinence with our study, however, their mean age was higher than our study (53.7 \pm 10.9 years) and all subjects had diabetes mellitus.

The prevalence of fecal incontinence was reported diversely, from $1 \%$ to $36 \%$. $^{1-5}$ The variability depends on the study population, study setting and definition of fecal incontinence. One study conducted in adults older than 40 years has found the fecal incontinence in $1.4 \% .{ }^{19}$ In this study, fecal incontinence was defined by its frequency as several times a month, but in another study subjects aged 70 and older showed the prevalence of $17 \%$, defining its frequency as once or more during the past few months. ${ }^{20}$ In other 2 different studies conducted in primary care centers in Spain and USA in 2010, each of their prevalence of fecal incontinence was appeared as $10.8 \%$ and $36.2 \%$, respectively. ${ }^{4,21}$ In the Spanish study, their definition of fecal incontinence only included the uncontrolled passage of stool within the recent 4 weeks, ${ }^{21}$ but in the American study, fecal incontinence was much broadly defined as any accident of fecal leaking within the recent 3 months.

The development of fecal incontinence is known to be multi-factorial. From previous studies, several contributing factors have been identified to be including structural abnormalities like obstetric injury, hemorrhoid, functional gastrointestinal disorders such as IBS and other several physically disabled conditions, disordered cognitive function, and also medications. ${ }^{1-8,19}$ Among those factors, old age, female sex, watery stool consistency, diabetes and depression have been widely considered to be predictive factors of fecal incontinence. ${ }^{5,7,18}$

It is commonly considered that age-related thinning of the external sphincter muscles may contribute to higher rates of fecal incontinence in older individuals, ${ }^{22}$ however the exact mechanisms that could explain the effect of age on fecal incontinence are unknown. ${ }^{1}$ In female, obstetrical injuries and IBS are considered as the reasons of frequent fecal incontinence..$^{5}$ In the present study, female subjects have also shown more frequent fecal incontinence than male subjects, but did not reach the significant difference. We did not evaluate the detailed obstetrical history, but there was no significant female predominance of IBS in our study. It might be related with the relatively lower prevalence of female fecal incontinence than that of Western society.

In the present study, stool consistency, especially watery stool and functional diarrhea, was a strong predictor for fecal incontinence. The previous studies showed that diarrhea symptoms, especially urgency to be a risk factor for fecal incontinence. ${ }^{2}$ Various symptoms suggestive of constipation such as incomplete evacuation, anal blockage are also risk factors for fecal incontinence. Previous study showed that $40 \%$ of subjects having incomplete evacuation suffered from fecal incontinence and $15 \%$ of them developed fecal incontinence in the following 9 years. ${ }^{3}$

Depression is well known risk factor for fecal incontinence. Recently, several studies revealed the association between depression and fecal incontinence. ${ }^{7}$ The relationship may be bidirectional. Dysregulation of central nervous system in patients with depression may affect the gastrointestinal motor and sensory function. And recurrent spillage of fecal material could cause or aggravate the depressed mood, inversely. Several studies which reported fecal incontinence affected QOL and self esteem support these observation. ${ }^{9-10}$

Diarrhea has been frequently noted in patients with diabetes, and those who have had previous experience of cholecystectomy. ${ }^{23}$ Therefore, in some studies, diabetes and cholecystectomy have also been considered as risk factors for fecal incontinence. ${ }^{17,23}$ In addition, diabetes might also contribute to the development of fecal incontinence through neurologic and micro-vascular pathways. Theses mechanisms may be related with the damage of innervations or muscular dysfunction of rectum and pelvic floor. ${ }^{4,6,23}$ However, in this study, both cholecystectomy and diabetes were proven not to be independent predictors for fecal incontinence, though watery stools and IBS showed the significant correlations with fecal incontinence. Obesity might also contribute to fecal incontinence by increasing the intra-abdominal pressures, ${ }^{23}$ but it did not show any correlation with fecal incontinence in the present study.

Our study has several strengths. The present study was not restricted to specific subgroup aside from age, gender or specific conditions, such as selected aged subjects, having diabetes or staying at nursing home. Second, the information was collected with a validated, self-reporting questionnaires. People who have fecal incontinence might have a tendency of underreporting their 
symptoms

We do also have the potential limitations. This work is a cross sectional study, therefore, the causal relation is not clear. For example, depression is known as a predictor for fecal incontinence, but at the same time, fecal incontinence might provoke or aggravate the depressed mood, too. Second, we enrolled subjects from Health Promotion Center, therefore, the study subjects might not have generalizability. Two-thirds of the study subjects visited the Health Promotion Center to undergo a group medical examination once a year or biannually and only ont-third paid for by themselves. Therefore, some proportion of subjects who were enrolled in the present study had different characters from general population. The previous study in Korea demonstrated that the study of health screening population had higher income and educational status compared with general Korean population. However, other factor such as BMI, smoking, alcohol habit or co-morbidities were comparable to the background population. ${ }^{24,25}$ In the present study, these factors were not significantly different between cases and controls. When we adjusted the prevalence of fecal incontinence with age and gender to census in 2009 (http:/www.census.go.kr/hcensus/index.jsp), the prevalence of fecal incontinence was $7.0 \%$.

As the proportion of older people in the community is increasing, fecal incontinence might become an important issue related with socioeconomic burden. Our results confirmed that the bowel pattern is one of the most important risk factor for fecal incontinence. We could say that fecal incontinence might be treated by removing factors that could affect bowel motility, such as inflammations, use of the laxatives, and by encouraging patients to take enough fiber or pills like loperamide or serotonin receptor antagonists. ${ }^{26,27}$ Moreover, cognitive or behavioral therapies might be helpful for controlling the fecal incontinence in subset of patients. ${ }^{21,26,27}$

In conclusion, most of the fecal incontinence was mild form and was related with watery or loose stool consistency. Therefore, substantial portion of subjects with fecal incontinence might be easily tolerable with controlling stool consistency and bowel motility by diet or medication. The proper patients' education programs might be needed because most patients had limited knowledge about fecal incontinence as irreversible or devastating condition. Physicians should raise the awareness on fecal incontinence and provide competent counseling and treatment to patients.

\section{References}

1. Whitehead WE, Borrud L, Goode PS, et al. Fecal incontinence in US adults: epidemiology and risk factors. Gastroenterology 2009; 137:512-517.

2. Kalanta JS, Howell S, Talley NJ. Prevalence of faecal incontinence and associated risk factors: an underdiagnosed problem in the Australian community? Med J Aust 2002;176:54-57.

3. Rey E, Choung RS, Schleck CD, Zinsmeister AR, Locke GR 3rd, Talley NJ. Onset and risk factors for fecal incontinence in a US community. Am J Gastroenterol 2010;105:412-419.

4. Dunivan GC, Heymen S, Palsson OS, et al. Fecal incontinence in primary care: prevalence, diagnosis, and health care utilization. Am J Obstet Gynecol 2010;202:493.e1-e6.

5. Nelson R, Norton N, Cautley E, Furner S. Community-based prevalence of anal incontinence. JAMA 1995;274:559-561.

6. Varma MG, Brown JS, Creasman JM, et al. Fecal incontinence in females older than aged 40 years: who is at risk? Dis Colon Rectum 2006;49:841-851.

7. Melville JL, Fan MY, Newton K, Fenner D. Fecal incontinence in US women: a population-based study. Am J Obstet Gynecol 2005; 193:2071-2076.

8. Bharucha AE, Zinsmeister AR, Locke GR, et al. Prevalence and burden of fecal incontinence: a population-based study in women. Gastroenterology 2005;129:42-49.

9. Kamm MA. Faecal incontinence. BMJ 2003;327:1299-1300.

10. Bartlett L, Nowak M, Ho YH. Impact of fecal incontinence on quality of life. World J Gastroenterol 2009;15:3276-3282.

11. Johanson JF, Lafferty J. Epidemiology of fecal incontinence: the silent affliction. Am J Gastroenterol 1996;91:33-36.

12. Talley NJ, Phillips SF, Melton J 3rd, Wiltgen C, Zinsmeister AR. A patient questionnaire to identify bowel disease. Ann Intern Med 1989;111:671-674.

13. Song HJ, Jung HK, Yeom HJ, et al. Reliability and validity of Korean bowel disease questionnaire and prevalence of functional gastrointestinal disorders in Korea. Gut 2009;58(suppl 1):A112.

14. Attanasio V, Andrasik F, Blanchard EB, Arena JG. Psychometric properties of the SUNY revision of the psychosomatic symptom checklist. J Behav Med 1984;7:247-257.

15. Drossman DA. The functional gastrointestinal disorders and the Rome III process. Gastroenterology 2006;130:1377-1390.

16. Huxley R, James WP, Barzi F, et al. Ethnic comparisons of the cross-sectional relationships between measures of body size with diabetes and hypertension. Obes Rev 2008;9(suppl 1):53-61.

17. Joh HK, Seong MK, Oh SW. Fecal incontinence in elderly Koreans. J Am Geriatr Soc 2010;58:116-121.

18. Oh JH, Choi MG, Kang MI, et al. The prevalence of gastrointestinal symptoms in patients with non insulin dependent diabetes mellitus. Korean J Intern Med 2009;24:309-317.

19. Perry S, Shaw C, McGrother C, et al. Prevalence of faecal incontinence in adults aged 40 years or more living in the community. Gut 2002;50:480-484.

20. Resnick NM, Beckett LA, Branch LG, Scherr PA, Wetle T. Short term variability of self report of incontinence in older persons. J Am Geriatr Soc 1994;42:202-207. 
21. Parés D, Vial M, Bohle B, et al. Prevalence of fecal incontinence and analysis of its impact on quality of life and mental health. Colorectal Dis 2011;13:899-905.

22. Frudinger A, Halligan S, Bartram CI, Price AB, Kamm MA, Winter R. Female anal sphincter: age-related differences in asymptomatic volunteers with high-frequency endoanal US. Radiology 2002;224:417-423.

23. Goode PS, Burgio KL, Halli AD, et al. Prevalence and correlates of fecal incontinence in community dwelling older adults. J Am Geriatr Soc 2005;53:629-635.

24. Kim N, Lee SW, Cho SI, et al. The prevalence of and risk factors for erosive oesophagitis and non-erosive reflux disease: a nationwide multicentre prospective study in Korea. Aliment Pharmacol Ther 2008;27:173-185.

25. Kim JH, Hwang JK, Kim J, et al. Endoscopic findings around the gastroesophageal junction: an experience from a tertiary hospital in Korea. Korean J Intern Med 2008;23:127-133.

26. Markland AD, Richter HE, Burgio KL, Wheeler TL 2nd, Redden DT, Goode PS. Outcomes of combination treatment of fecal incontinence in women. Am J Obstet Gynecol 2008;199:699.e1-7.

27. Norton C, Thomas L, Hill J, Guideline Development Group. Management of faecal incontinence in adults: summary of NICE guideline. BMJ 2007;334:1370-1371 